Proceedings of the Institute of Mathematics and Mechanics,

National Academy of Sciences of Azerbaijan

Volume 46, Number 1, 2020, Pages 56-67

https://doi.org/10.29228/proc. 17

\title{
THE SHEAVES REPRESENTATION OF HAUSDORFF SPECTRA
}

\author{
EUGENY I. SMIRNOV, SERGEY A. TIKHOMIROV, AND ELENA A. ZUBOVA
}

\begin{abstract}
We introduce new concepts of functional analysis: Hausdorff spectrum and Hausdorff limit or H-limit of Hausdorff spectrum of locally convex spaces with point of view using sheaves theory. Particular cases of regular $\mathrm{H}$-limit are projective and inductive limits of separated locally convex spaces. The class of H-spaces contains Fréchet spaces and is stable under the operations of forming countable inductive and projective limits, closed subspaces and factor-spaces. Besides, for $\mathrm{H}$-space the strengthened variant of the Closed Graph Theorem holds true. The space of germs of holomorphic functions on connected bounded subset will be provided with the topology (in general not separated) of uniform convergence on the compact subsets and with the locally convex topology of the H-limit. We also present an essentially new approach to the study of sheaves based on the notion of Hausdorff spectra associated with the presheaf.
\end{abstract}

\section{Introduction}

The study which was carried out in [12]-[13] of the derivatives of the projective limit functor acting from the category of countable inverse spectra with values in the category of locally convex spaces made it possible to resolve universally homomorphism questions about a given mapping in terms of the exactness of a certain complex in the abelian category of vector spaces. Later in [17] a broad generalization of the concepts of direct and inverse spectra of objects of an additive semiabelian category $\mathcal{G}$ was introduced: the concept of a Hausdorff spectrum, analogous to the $\delta s$-operation in descriptive set theory. This idea is fruitful even for algebraic topology, general algebra, category theory and the theory of generalized functions.

On the other hand, the issues related to different types of sheaves, including bundles, still attracts great interest and primarily in solving problems of algebraic geometry (about recent progress in this field see papers [1], [5]-[11], [18]-[19]). And it should be recognized that the concept of the spectrum of a reflexive sheaf of rank two on $\mathbb{P}^{3}$, introduced in [4] for the field of any characteristic, turned out to be very useful for such purposes. Such spectrum have a numerical nature.

2010 Mathematics Subject Classification. 46A13, $14 \mathrm{~F} 05$.

Key words and phrases. topology, spectrum, closed graph theorem, sheaves theory, homological methods, category. 
We present an essentially new approach to the study of sheaves by means of non-numerical spectra. This approach based on the notion of Hausdorff spectrum associated with the presheaf. In Sect. 2 we provide the preliminary background and introduce the concepts of Hausdorff spectrum and Hausdorff limit or H-limit of Hausdorff spectrum of locally convex spaces with point of view using sheaves theory. In Sect. 3 we prove main results of this paper - Theorems 3.1-3.3, which lay the foundation for the intriguing direction on a joint of functional analysis and algebraic geometry.

\section{Hausdorff Spectrum and Hausdorff Limit}

The construction of Hausdorff spectra $\mathcal{X}=\left\{X_{s}, \mathbf{F}, h_{s^{\prime} s}\right\}$ is achieved by successive standard extension of a small category of indices $\Omega$. The category $\mathcal{H}$ of Hausdorff spectra turns out to be additive and semiabelian under a suitable definition of mapping of spectra. In particular, $\mathcal{H}$ contains V. P. Palamodov's category of countable inverse spectra with values in the category $T L C$ of locally convex spaces [12].

The $H$-limit of a Hausdorff spectrum in the category $T L C$ generalizes the concepts of projective and inductive limits and is defined by the action of the functor Haus : $\mathcal{H} \longrightarrow T L C$. The class of $H$-spaces is defined by the action of the functor Haus on the countable Hausdorff spectra over the category of Banach spaces; the Closed Graph Theorem holds for its objects [16] and it contains the category of Fréchet spaces and the categories of spaces due to De Wilde [2], D. A. Rajkov [14] and P. P. Zabrejko - E. I. Smirnov [20].

Further, we recall certain definitions and results which were brought into the discussion in [14], [15], [17], [20].

Definition 2.1. Let $\Omega$ be a small category. By a directed class in the category we mean a subcategory satisfying the following properties:

(i) no more than one morphism is defined between any two objects;

(ii) for any objects $a, b$ there exists an object $c$ such that $a \rightarrow c$ and $b \rightarrow c$.

Definition 2.2. Let $A$ be some category and $s$ denotes the object of category $A$ (if $Q \in \Omega$ and $a, b \in Q$ we will denote the corresponding morphisms of category $\Omega$ by $a \stackrel{Q}{\longrightarrow} b)$. We shall call the category $B$ with objects $S$, where $S$ is a subcategory of $A$, a standard extension of the category $A$ if the following conditions are satisfied:

1. $A$ is a complete subcategory of $B$;

2. Morphism $\omega_{S S^{\prime}}: S^{\prime} \rightarrow S$ of the category $B$ is defined by the collection of morphisms $\omega_{s s^{\prime}}: s^{\prime} \longrightarrow s,\left(s^{\prime} \stackrel{\omega_{s s^{\prime}}}{\longrightarrow} s\right)$ of the category $A$ such that

(a) for every $s^{\prime} \in S^{\prime}$ there exists $s \in S$ such that $s^{\prime} \stackrel{\omega_{s s^{\prime}}}{\longrightarrow} s$;

(b) if $s^{\prime} \stackrel{\omega_{s s^{\prime}}}{\longrightarrow} s, p^{\prime} \stackrel{\omega_{p p^{\prime}}}{\longrightarrow} p, s \stackrel{\omega_{p s}^{S}}{\longrightarrow} p$, then there exists a morphism $s^{\prime} \stackrel{\omega_{p s}^{S^{\prime}}}{\longrightarrow} p^{\prime}$ and the following diagram is commutative:

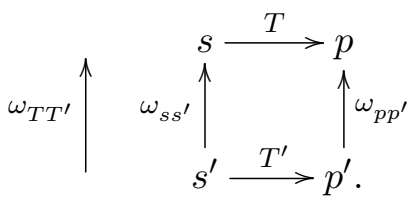


Definition 2.3. We will establish the successive standard extensions of categories

$$
\Omega(s) \subset \boldsymbol{B}(T) \subset \Sigma(F) \rightarrow \Sigma^{0}(F) \subset \boldsymbol{D}(\boldsymbol{F}),
$$

where $T \subset \Omega$ denotes directed classes of objects $s \in \Omega$, coinsides as object of category $\boldsymbol{B} ; F, F \in \boldsymbol{B}$ denotes filter bases of sets $T \in \boldsymbol{B}$, considered as objects of category $\Sigma$, and $\boldsymbol{F}, \boldsymbol{F} \subset \Sigma$ denotes directed classes of objects $F \in \Sigma$ of the dual category $\Sigma^{0}$, considered as objects of category $\boldsymbol{D}$. We shall say that such classes $\boldsymbol{F}$ are admissible for $\Omega$.

Let us put $|F|=\cup_{T \in \mathbf{F}} T,|\mathbf{F}|=\cup_{F \in \mathbf{F}} T$, so that $|F| \subset \Omega$ and $|\mathbf{F}| \subset \Omega$. The most characteristic constructions connected with Hausdorff spectra use in the role of the small category $\Omega=\operatorname{OrdI}$, where $I$ is a partially ordered set of indices, considered as category.

A diagram explaining the nature of the indexing is given below:

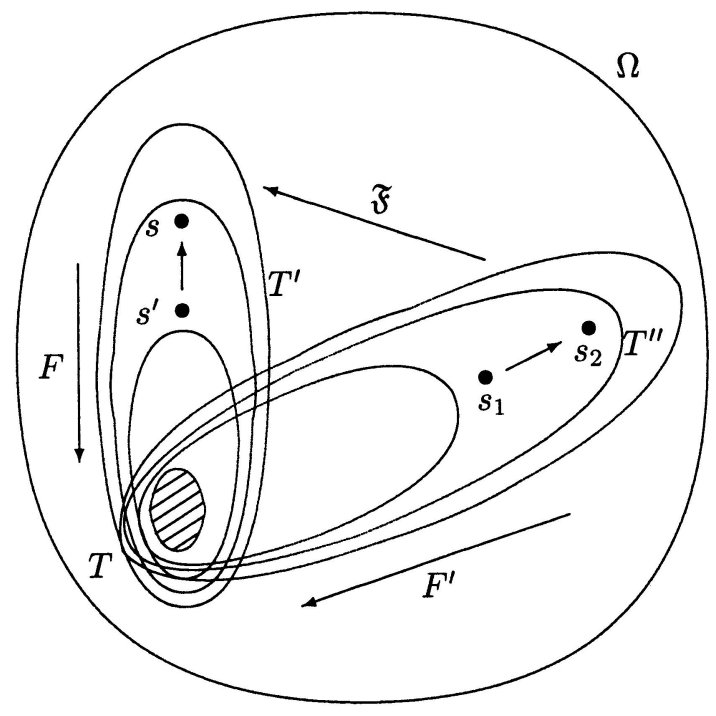

Definition 2.4. Let $\mathcal{G}$ be some category. We shall call a covariant functor $H_{\mathbf{F}}$ : $\Omega \rightarrow G$ a Hausdorff spectrum functor if $\Omega=|\mathbf{F}|$ for some admissible class $\mathbf{F} \in D$. If $\mathbf{F}=|\mathbf{F}|$ then $H_{\mathbf{F}}$ is a functor of the direct spectrum, while if $\mathbf{F}=|\mathbf{F}|$ (that is, $\mathbf{F}$ consists of a single element $|F|=|\mathbf{F}|$ ) then $H_{\mathbf{F}}$ is a functor of the inverse spectrum. If $H_{\mathbf{F}}$ is an admissible class for $\Omega$ and the functor $h_{\mathbf{F}}$ :

$|\mathbf{F}| \rightarrow \mathcal{G}: s \mapsto X_{s},\left(s^{\prime} \stackrel{\omega_{s s^{\prime}}}{\longrightarrow} s\right) \mapsto\left(X_{s} \rightarrow X_{s^{\prime}}\right),\left(F^{\prime} \stackrel{\omega_{F F^{\prime}}}{\longrightarrow} F\right) \mapsto\left(\left(X_{s}\right)_{s \in|F|} \rightarrow\left(X_{s^{\prime}}\right)_{s^{\prime} \in\left|F^{\prime}\right|}\right)$

is injective on objects and morphisms (in the set-theoretic sense), then there exists a directed class

$$
\left(\left(X_{s}\right)_{s \in|F|}, q_{F F^{\prime}}\right)_{F, F^{\prime}} \in \mathbf{F}
$$

of classes $\left(X_{s}, h_{s^{\prime} s}\right)_{s, s^{\prime} \in|F|}(F \in \mathbf{F})$ which are directed in the dual category $\mathcal{G}^{0}$ and which satisfy the following conditions:

1. $X_{s} \stackrel{h_{s^{\prime}}}{\longrightarrow} X_{s^{\prime}}$ is determined and fixed if and only if the morphism $s^{\prime} \stackrel{\omega_{s s^{\prime}}}{\longrightarrow} s$ is determined and then $h_{s^{\prime} s}: X_{s} \longrightarrow X_{s^{\prime}}$ is the only morphism. 
2. The diagram

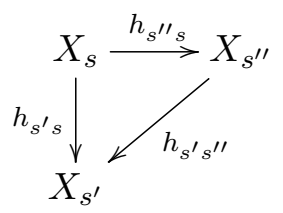

is commutative for all $s^{\prime \prime} \stackrel{\omega_{s^{\prime} s^{\prime \prime}}}{\longrightarrow} s^{\prime} \stackrel{\omega_{s s^{\prime}}}{\longrightarrow} s$.

3. If $\left(X_{s}\right)_{s \in|F|} \stackrel{q_{F^{\prime} F}}{\longrightarrow}\left(X_{s^{\prime}}\right)_{s^{\prime} \in\left|F^{\prime}\right|}$, then for each $X_{s^{\prime}}\left(s^{\prime} \in\left|F^{\prime}\right|\right)$ there exists a unique morphism $h_{s^{\prime} s}: X_{s} \rightarrow X_{s^{\prime}}(s \in|F|)$. The collection of morphisms $h_{s^{\prime} s}$ $\left(s^{\prime} \in\left|F^{\prime}\right|\right)$ defines the morphism $q_{F^{\prime} F}$ so that we will write $q_{F^{\prime} F}=\left(h_{s^{\prime} s}\right)_{F^{\prime} F}$. Each set $F \in \mathbf{F}$ is a filter base of subsets $T \subset|F|$ and moreover for each $T \in F$ the class $\left(X_{s}, h_{s^{\prime} s}\right)_{T}$ is directed in the category $\mathcal{G}^{0}$.

We will call a class $\left(X_{s}, h_{s^{\prime} s}\right)_{s, s^{\prime} \in|F|}$ satisfying conditions (1)-(3) a Hausdorff spectrum over the category $\mathcal{G}$ and we will denote it by $\left\{X_{s}, \mathbf{F}, h_{s^{\prime} s}\right\}$.

The direct and inverse spectra of a family of objects are particular cases of Hausdorff spectra - it suffices to put $\mathbf{F}=|\mathbf{F}|, h_{s^{\prime} s}=q_{F^{\prime} F}$ in the direct case and $\mathbf{F}=\{|\mathbf{F}|\}, h_{s^{\prime} s}: X_{s} \rightarrow X_{s^{\prime}}\left(s^{\prime} \rightarrow s\right), q_{F^{\prime} F}=i_{|F|}=i_{|\mathbf{F}|}$ in the inverse case.

Under a suitable definition of spectral mapping (see the structure of the category $\mathrm{D}(\mathrm{F})$ ) the set of Hausdorff spectra over $\mathbf{G}$ forms a category which we denote by Spect $\mathbf{G}$. If $\mathbf{X}=\left\{X_{s}, \mathbf{F}, h_{s^{\prime} s}\right\}, \mathbf{Y}=\left\{Y_{p}, \mathbf{F}^{1}, h_{p^{\prime} p}\right\}$ are objects from Spect $\mathbf{G}$, then we shall say that two Hausdorff spectrum mappings $\omega_{\mathbf{Y X}}: \mathbf{X} \rightarrow \mathbf{Y}$ and $\omega_{\mathbf{Y X}}^{\prime}: \mathbf{X} \rightarrow \mathbf{Y}$ are equivalent if for any $F \in \mathbf{F}$ there exists $F^{*} \in \mathbf{F}^{1}$ such that the diagram

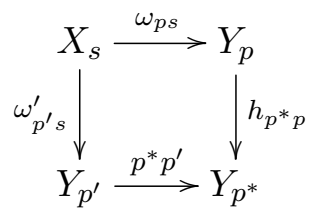

is commutative for any $p^{*} \in\left|F^{*}\right|$.

Now let us consider a new category $\mathbf{H}(\mathbf{G})$ whose objects are the objects of the category Spect $\mathbf{G}$, but the set $\operatorname{Hom}_{\mathbf{H}}(\mathbf{X}, \mathbf{Y})$ is formed by the equivalence classes of mappings $\omega_{\mathbf{Y X}}: \mathbf{X} \rightarrow \mathbf{Y}$. We shall denote such classes by $\left\|\omega_{\mathbf{Y} \mathbf{X}}\right\|$.

For any objects $\mathbf{X}, \mathbf{Y}, \mathbf{Z} \in \mathbf{H}$ the law of composition defines a bilinear mapping

$$
\operatorname{Hom}_{\mathbf{H}}(\mathbf{X}, \mathbf{Y}) \times \operatorname{Hom}_{\mathbf{H}}(\mathbf{Y}, \mathbf{Z}) \longrightarrow \operatorname{Hom}_{\mathbf{H}}(\mathbf{X}, \mathbf{Z})
$$

$\left(\operatorname{Hom}_{\mathbf{H}}(\mathbf{X}, \mathbf{Y})\right.$ is an abelian group).

Definition 2.5. Let $\mathbf{X}=\left\{X_{s}, \mathbf{F}, h_{s^{\prime} s}\right\}$ be a Hausdorff spectrum over the category G. We will call an object $Z$ of the category $\mathbf{G}$ a categorical $H$-limit of the Hausdorff spectrum $\boldsymbol{X}$ over $\boldsymbol{G}$ if for any objects $A, B \in \mathbf{G}$ and mappings of spectra

$$
A \stackrel{a}{\longrightarrow} \mathbf{X} \stackrel{b}{\longrightarrow} B
$$

there exists a unique sequence in $\mathbf{G}$

$$
A \stackrel{a}{\longrightarrow} \mathbf{Z} \stackrel{b}{\longrightarrow} B
$$


such that the diagram

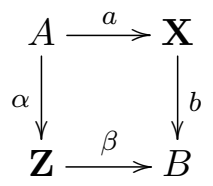

is commutative in the category Spect $\mathbf{G}$.

The concepts of projective and inductive limits over the category $\mathbf{G}$ are special cases of categorical $H$-limits. For example, let $\mathbf{X}$ be an inverse spectrum of objects from $\mathbf{G}$. Then the previous diagram holds and moreover any object $X_{s}$ from $\mathbf{X}$ can be taken for $B \in \mathbf{G}$ with the identity morphism $b_{s}: X_{s} \longrightarrow X_{s}$ forming the spectral mapping $b^{s}: \mathbf{X} \longrightarrow X_{s}(s \in|\mathbf{F}|)$. Thus the diagram

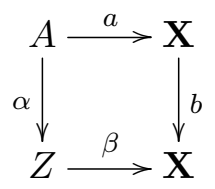

is commutative, where $b=\left(b^{s}\right), \beta=\left(\beta^{s}\right), \beta^{s}: Z \longrightarrow X_{s}(s \in \mathbf{F})$ and $b$ is the identity morphism of the category $\mathbf{G}$. Therefore the diagram

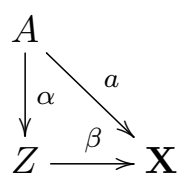

is commutative for any object $A \in \mathbf{G}$.

The categorical $H$-limit of a Hausdorff spectrum (the functor Hom) exists in any semiabelian category $\mathbf{G}$ with direct sums and products (for example, the category of vector spaces $L$, the category $T L G$ of topological vector groups, the category $T L C$ of locally convex spaces).

Let $\Omega$ be a countable set and $X=X_{s}, \mathbf{F}, h_{s^{\prime} s}$ a regular Hausdorff spectrum in the category $T L C$; such a spectrum is said to be countable. A continuous linear image in the category $T L C$ of an $H$-limit of Banach spaces $X_{s}(s \in|\mathbf{F}|)$ is called an $H$-space. The class of $H$-spaces contains the Fréchet spaces and is stable with respect to the operations of passage to countable inductive and projective limits, closed subspaces and factor spaces. Moreover, a strengthened variant of the Closed Graph Theorem holds for $H$-spaces. The class of $H$-spaces is the broadest of all the analogous classes known at this time, namely those of Rajkov, De Wilde, Hakamura, Zabrejko-Smirnov. A countable separated regular $H$-limit of a Hausdorff spectrum of $H$-spaces in the category $T L C$ is an $H$-space [2].

\section{Hausdorff Spectra and Sheaves}

Let $\left\{\mathcal{S}_{U}, \rho_{U V}\right\}$ be a presheaf of abelian groups over a topological space $\mathcal{D}, \Omega$ a nonempty partially ordered set and $\mathbf{F}$ an admissible class for $\Omega$ (we may assume without loss of generality that $\Omega=|\mathbf{F}|)$. Let us denote by $\hat{H}(\mathcal{S})$ a covariant functor from $\operatorname{Ord} \Omega$ to $\operatorname{Ord} \mathcal{U}$, where $\mathcal{U}$ is a base of open sets in $\mathcal{D}$, and by $\check{H}(\mathcal{S})$ a contravariant functor from $\operatorname{Ord} \mathcal{U}$ to the category of abelian groups so that an abelian group $\mathcal{S}_{U}$ is defined for each $U \in \mathcal{U}$ and a homomorphism $\rho_{U V}: \mathcal{S}_{U} \rightarrow \mathcal{S}_{V}$ 
is defined for each pair $U \subset V$. Then $H=\check{H}(\mathcal{S}) \circ \hat{H}(\mathcal{S})$ is a contravariant functor of the Hausdorff spectrum $\mathcal{X}(\mathcal{S})=\left\{\mathcal{S}_{U_{s}}, \mathbf{F}, \rho_{U_{s^{\prime}} U_{s}}\right\}$.

Definition 3.1. We will call functor $H$ the Hausdorff spectrum associated with the presheaf $\left\{\mathcal{S}_{U}, \rho_{U V}\right\}$.

Let $X$ be the $H$-limit of the Hausdorff spectrum $\mathcal{X}(\mathcal{S})$ in the category of abelian groups and let

$$
A=\bigcap_{F \in \mathbf{F}} \bigcup_{s \in|F|} U_{s} .
$$

Theorem 3.1. Let $\mathcal{S}$ be the sheaf of germs of holomorphic functions on an open set $\mathcal{D} \subset \mathbb{C}^{n}$, associated with the presheaf $\left\{\mathcal{S}_{U}, \rho_{U V}\right\}$, and let $\mathcal{X}(\mathcal{S})=$ $\left\{\mathcal{S}_{U_{s}}, \boldsymbol{F}, \rho_{U_{s^{\prime}} U_{s}}\right\}$ be the associated true Hausdorff spectrum. Then the H-limit of the Hausdorff spectrum $\mathcal{X}(\mathcal{S})$ is isomorphic to the vector space of sections $\Gamma(A, \mathcal{S})$ of the sheaf $\mathcal{S}$ over the set $A$.

Proof. By the conditions relating to $\left\{\mathcal{S}_{U}, \rho_{U V}\right\}$, we may put $\mathcal{S}_{U}=\Gamma(U, \mathcal{S})(U \in$ $\mathcal{U})$. Further, let

so that

$$
X=\overleftrightarrow{\lim }_{\mathbf{F}} \rho_{U_{s^{\prime}} U_{s}} \Gamma\left(U_{s}, \mathcal{S}\right)
$$

$$
X=\bigcup_{F \in \mathbf{F}} \bigcap_{T \in F} \psi\left(V_{F}^{T}\right)
$$

If $x \in X$, there exists $F \in \mathbf{F}$ such that $x \in \psi\left(V_{F}^{T}\right)(T \in F)$, that is to say, there exists a selection

$$
\xi(T)=\left(f_{s}^{T}\right)_{s \in|F|}
$$

such that $\psi\left(f_{s}^{T}\right)=x$ for each $T \in F$. For any $U \in \mathcal{U}_{z}(z \in \mathcal{D})$ the homomorphism $\rho_{z U}: \Gamma(U, \mathcal{S}) \rightarrow \mathcal{S}_{z}$ generates for $f \in \Gamma(U, \mathcal{S})$ the set of points

$$
\rho_{U}(f)=\bigcup_{z \in U} \rho_{z U}(f) \subset \mathcal{S},
$$

therefore let us put

$$
\rho_{x}^{T}=\bigcup_{s \in T} \rho_{U_{s}}\left(f_{s}^{T}\right) ;
$$

it is clear that $\rho_{x}^{T}$ generates the section $f^{T}$ on the open set $U_{T}=\bigcup_{s \in T} U_{s}$, since the correspondence

$$
z \in U_{T} \stackrel{f^{T}}{\longmapsto} \rho_{x}^{T} \cap \mathcal{S}_{z} \subset \mathcal{S}
$$

is single-valued and continuous. Moreover, if $\rho_{U V}: \rho_{V}(g) \mapsto \rho_{U}(f)$, then $\rho_{U}(f) \subset$ $\rho_{V}(g)$, so let us put

$$
\rho_{x}^{\xi}=\bigcup_{F^{*} \succ F} \bigcup_{s^{*} \in\left|F^{*}\right|, s \in T} \rho_{U_{s^{*}} U_{s}}\left(\rho_{U_{s}}\left(f_{s}^{T}\right)\right),
$$

where necessarily

$$
\rho_{U_{s^{*}} U_{s}}\left(\rho_{U_{s}}\left(f_{s}^{T}\right)\right)=\rho_{U_{s^{*}} U_{s}}\left(\rho_{U_{s}}\left(f_{s}^{T^{\prime}}\right)\right) \quad\left(T, T^{\prime} \in F\right) .
$$

Let us put

$$
U_{\rho_{x}}=\bigcap_{\xi} U_{\rho_{x}^{\xi}}, \quad \text { where } \quad U_{\rho_{x}^{\xi}} \subset \bigcup_{s \in|F|} U_{s}
$$


in this connection we have in particular,

$$
\rho_{U_{s}}\left(f_{s}^{T}\right) \cap \rho_{U_{s}}\left(f_{s}^{T^{\prime}}\right) \supset \rho_{U_{s} U_{s}}\left(\rho_{U_{s}}\left(f_{s}^{T}\right)\right) .
$$

It is also clear that for each $\xi$ the correspondence

$$
z \in U_{\rho_{x}^{\xi}} \mapsto \rho_{x}^{\xi} \cap \mathcal{S}_{z}
$$

is single-valued and continuous. Although, in general, it is not guaranteed that $U_{\rho_{x}} \neq \emptyset$, we will show nevertheless that $U_{\rho_{x}} \supset A$ under the conditions of the Theorem, specifically because the $H$-limit of the Hausdorff spectrum $\mathcal{X}(\mathcal{S})$ is true. Let the selection $\xi(T)=\left(f_{s}^{T}\right)_{s \in|F|}(T \in F)$ generating the element $x \in X$ be fixed. Then because the Hausdorff spectrum $\mathcal{X}(\mathcal{S})$ is true we may assume that $f_{s}^{T_{1}}=f_{s}^{T_{2}}\left(s \in T_{1} \cap T_{2}\right)$ and, consequently, there exists $\xi=\left(f_{s}\right)_{s \in|F|} \in \bigcap_{T \in F} V_{F}^{T}$ such that

$$
x \in \psi\left(\left(f_{s}\right)_{|F|}\right) \quad \text { and } \quad f_{s^{\prime}}=\rho_{U_{s^{\prime}} U_{s}}\left(f_{s}\right) \quad\left(s, s^{\prime} \in|F|\right) .
$$

It is clear that $\rho_{x}^{\xi}=\bigcup_{s \in|F|} \rho_{U_{s^{\prime}} U_{s}}\left(f_{s}\right)$. Now let $z \in A$. Then $z \in U_{\rho_{x}^{\xi}}$ for any $\xi(F)$ $(F \in \mathbf{F})$ and, moreover,

$$
\rho_{x}^{\xi}(z)=\rho_{x}^{\xi} \cap \mathcal{S}_{z}=\rho_{z U_{s}}\left(f_{s}\right) \quad \text { for } \quad z \in U_{s} \quad(s \in|F|) .
$$

Let us show that $\rho_{x}^{\xi}(z)=\rho_{x}^{\xi^{\prime}}(z)$ for any $\xi, \xi^{\prime}$. In fact, let $\xi=\left(f_{s}\right)_{|F|}, \xi^{\prime}=\left(f_{s^{\prime}}^{\prime}\right)_{\left|F^{\prime}\right|}$ and $x=\psi(\xi), x^{\prime}=\psi\left(\xi^{\prime}\right)$. Since $\xi \sim \xi^{\prime}$, there exists $F^{*} \in \mathbf{F}$, where $F^{*} \succ F^{\prime}$ and $F^{*} \succ F^{\prime}$, such that for each $T^{*} \in F^{*}$ we can find $T \in F$ and $T^{\prime} \in F^{\prime}$ such that

$$
\omega_{T T^{*}}: T^{*} \rightarrow T, \quad \omega_{T^{\prime} T^{*}}: T^{*} \rightarrow T^{\prime} \quad \text { and } \quad \rho_{U_{s^{*}} U_{s}}\left(f_{s}\right)=\rho_{U_{s^{*}} U_{s^{\prime}}}\left(f_{s^{\prime}}^{\prime}\right),
$$

where $s^{*} \in T^{*}$. However, $z \in \bigcup_{s^{*} \in\left|F^{*}\right|} U_{s^{*}}$, and so it remains to choose $s_{0}^{*} \in\left|F^{*}\right|$, such that

$$
z \in U_{s_{0}^{*}} \quad \text { and } \quad \rho_{z U_{s}}\left(f_{s}\right)=\rho_{z U_{s^{\prime}}}\left(f_{s^{\prime}}^{\prime}\right) \quad\left(s^{*} \rightarrow s, s^{*} \rightarrow s^{\prime}\right) .
$$

Thus $z \in U_{\rho_{x}}$. Furthermore, let us put $x(z)=\left.\rho_{x}^{\xi}(z)\right|_{A}$, so that $x(z)$ is a section of $\mathcal{S}$ on $A, x(z) \in \Gamma(A, \mathcal{S})$. In this way we have constructed a morphism $\mathcal{H}$ : $X \rightarrow \Gamma(A, \mathcal{S})$. Given $f_{A}=\mathcal{H}(x), f_{A}=\mathcal{H}(y)$, let us prove that $x=y$. In fact, at each point $z \in A$ there exists an open ball $B(z, \epsilon)$ of the local homeomorphism $\pi: \mathcal{S} \rightarrow \mathcal{D}$ at the point $f_{A}(z)$. Let us put $U=\bigcup_{z \in A} B(z, \epsilon / 2)$ and determine the section $f_{z} \in \Gamma(B(z, \epsilon / 2), \mathcal{S})$ passing through the point $s=f_{A}(z) \in \mathcal{S}$ such that

$$
\left.f_{z}\right|_{A}=\left.f_{A}\right|_{B(z, \epsilon / 2)}
$$

(we note that $\epsilon=\epsilon(z)$ ). Let

$$
B_{i j}=B\left(z_{i}, \epsilon_{i} / 2\right) \cap B\left(z_{j}, \epsilon_{j} / 2\right), \quad B_{i j} \cap A \neq \emptyset, \quad z_{0} \in B_{i j} \cap A
$$

for some $z_{i}, z_{j} \in A$. Then $f_{z_{i}}\left(z_{0}\right)=f_{z_{j}}\left(z_{0}\right)$, and, consequently, there is an open ball $B_{0} \subset B\left(z_{0}, \epsilon_{0} / 2\right)$ of the local homeomorphism at the point $s_{0}=f_{z_{i}}\left(z_{0}\right)$ such that $B_{0} \subset B_{i j}$ and $\left.f_{z_{i}}\right|_{B_{0}}=\left.f_{z_{j}}\right|_{B_{0}}$. However, because of the isomorphism $\Gamma\left(B_{i j}, \mathcal{S}\right) \rightarrow \mathcal{S}_{B_{i j}}$ the holomorphic functions $f_{z_{i}}$ and $f_{z_{j}}$ coincide on the connected open set $B_{i j}[3]$. The last observation means that $\left.f_{z_{i}}\right|_{B_{i j}}=\left.f_{z_{j}}\right|_{B_{i j}}$. Now suppose that

$$
B_{i j} \cap A=\emptyset, \quad \text { but } \quad B_{i j}^{\prime}\left(\epsilon_{i}, \epsilon_{j}\right) \cap A \neq \emptyset, \quad z^{\prime} \in B_{i j}^{\prime} \cap A .
$$

Clearly, we will obtain by similar reasoning $\left.f_{z_{i}}^{\prime}\right|_{B_{i j}^{\prime}}=\left.f_{z_{j}}^{\prime}\right|_{B_{i j}^{\prime}}$. But we have $\left.f_{z_{i}}^{\prime}\right|_{B\left(z_{i}, \epsilon_{i} / 2\right)}=f_{z_{i}}$ and $\left.f_{z_{j}}^{\prime}\right|_{B\left(z_{j}, \epsilon_{j} / 2\right)}=f_{z_{j}}$, so that $\left.f_{z_{i}}\right|_{B_{i j}}=\left.f_{z_{j}}\right|_{B_{i j}}$ (in the case 
where $\left.B_{i j} \neq \emptyset\right)$. Now there remains the third possibility for $B_{i j} \neq \emptyset$, namely when $B_{i j}^{\prime} \cap A=\emptyset$. In this case the sections $f_{z_{i}}, f_{z_{j}}$ on $B_{i j}$ do not necessarily coincide, therefore let us put $M=\overline{\bigcup B_{i j}}$, where the bar denotes closure in $\mathbb{C}^{n}$ and the union is taken over all $B_{i j}$ of this third type. It is clear that $M \cap A=\emptyset$, since in the contrary case for $z^{*} \in M \cap A$ there would exist $B_{i j}^{*}$ of the third type such that $z^{*} \in B_{i j}^{* \prime}$, which is impossible by construction. Let us put $U\left(f_{A}\right)=U \backslash M$, so that $U\left(f_{A}\right) \supset A$ and $U\left(f_{A}\right)$ is an open subset of $\mathbb{C}^{n}$. Then there exists $f \in \Gamma\left(U\left(f_{A}\right), \mathcal{S}\right)$ such that $\left.f\right|_{A}=f_{A}$ and, moreover,

$$
\left.f\right|_{U\left(f_{A}\right) \cap B(z, \epsilon / 2)}=\left.f_{z}\right|_{U\left(f_{A}\right) \cap B(z, \epsilon / 2)} \quad(z \in A) ;
$$

also the section on $U\left(f_{A}\right)$ of $f$ with the property $\left.f\right|_{A}=f_{A}$ is uniquely determined (nevertheless, $\phi_{A}$, the corresponding holomorphic function on $A$, is extended holomorphically to $U\left(f_{A}\right)$ in a manner which, in general, is not unique).

Now if $\psi(\xi)=x, \psi(\eta)=y$, it follows from the fact that the family of open sets $\left\{\bigcup_{s \in|F|} U_{s}\right\}_{F \in \mathbf{F}}$ is fundamental for $A$ that there exists $F^{*} \in \mathbf{F}$ such that $U\left(f_{A}\right) \supset \bigcup_{F^{*}} U_{s^{*}}$, and moreover by construction

$$
\left.\rho_{x}^{\xi}\right|_{F^{*} U_{s^{*}}}=\left.\rho_{y}^{\eta}\right|_{\mathrm{U}^{*}} U_{s^{*}} .
$$

The last assertion means that $\xi \sim \eta$ and, consequently, $x=y$. Moreover, the fact that $\left\{\bigcup_{F} U_{s}\right\}_{F \in \mathbf{F}}$ is fundamental for $A$ and the constructions carried out above allow us to conclude that $\mathcal{H}: X \rightarrow \Gamma(A, \mathcal{S})$ is an isomorphism.

The absence of sufficiency restrictions on $|\mathbf{F}|$ in Theorem 3.1 allows us to apply it to any nonempty $A \subset \mathbb{C}^{n}$ : it is enough to take $\mathbf{F}=|\mathbf{F}|$ and $U_{s}(s \in$ F) a fundamental system of open sets containing $A$ (in general, of uncountable cardinality). The investigation of topological properties of $H$-limits in this case produces substantial difficulties, therefore the investigation of $\Gamma(A, \mathcal{S})$ with no more than countable $|\mathbf{F}|$ is of interest. It is clear that, for example, any closed bounded set $A \subset \mathbb{C}^{n}$ will be of this type, and the space of sections $\Gamma(A, \mathcal{S})$ is the inductive limit of the sequence of spaces $\Gamma\left(U_{s}, \mathcal{S}\right)(s \in|\mathbf{F}|)$. Furthermore, the corresponding Hausdorff spectrum $\left\{\Gamma\left(U_{s}, \mathcal{S}\right), \mathbf{F}, \rho_{U_{s^{\prime}} U_{s}}\right\}$ will be true in this case. In general, the Hausdorff spectrum $\mathcal{X}(\mathcal{S})$ will be true if all open sets $U_{s}(s \in|\mathbf{F}|)$ are connected. We recall that each space $\Gamma\left(U_{s}, \mathcal{S}\right)$ can be given the separated locally convex topology of uniform convergence on the compact subsets of $U_{s}$ $(s \in|\mathbf{F}|)$, under which it is a Fréchet space; we will denote this topology by $\tau_{s}$.

Theorem 3.2. Let $\mathcal{X}(\mathcal{S})=\left\{\Gamma\left(U_{s}, \mathcal{S}\right), F, \rho_{U_{s^{\prime}} U_{s}}\right\}$ be a true countable Hausdorff spectrum and suppose that $A=\bigcap_{\circ} \bigcup_{F} U_{s}$ has a countable fundamental system of compact sets, is connected and $\stackrel{\circ}{A} \neq \emptyset$. Then the $H$-limit

$$
\boldsymbol{X}=\overleftrightarrow{\lim }_{\boldsymbol{F}} \rho_{U_{s^{\prime}} U_{s}} \Gamma\left(U_{s}, \mathcal{S}\right)
$$

is a separated $H$-space in the topology $\tau^{*}$ and is continuously embedded in $\mathcal{O}_{A}$ $\left(\mathcal{O}_{A}\right.$ is the algebra of holomorphic functions on $\left.A\right)$.

Proof. First of all, by Theorem 3.1 we have the isomorphism $\mathcal{H}: X \rightarrow \Gamma(A, \mathcal{S})$; because of the connectedness of $A$ and the fact that $\stackrel{\circ}{A} \emptyset$ each holomorphic function on $A, \phi \in \mathcal{O}_{A}$, is generated by some holomorphic function on the open set $U(\phi)$; moreover, any two holomorphic functions $\phi_{1} \in \mathcal{O}_{U}$ and $\phi_{2} \in \mathcal{O}_{V}$ 
( $U \supset A, V \supset A$ ) which coincide on $A$ must coincide on a connected component of the intersection $U \cap V$ (see [3]), which also implies the isomorphism $\Gamma(A, \mathcal{S}) \equiv \mathcal{O}_{A}$. Since $A$ has a countable fundamental system of compact subsets

$$
K_{n} \quad(n=1,2, \ldots), \quad K_{1} \subset K_{2} \subset \ldots,
$$

on putting

$$
\|\phi\|_{n}=\max _{z \in K_{n}}|\phi(z)| \quad\left(\phi \in \mathcal{O}_{A}\right),
$$

we obtain a seminorm on $\mathcal{O}_{A}$ (or on $\Gamma(A, \mathcal{S})$, which is permissible according to the construction). Furthermore, on putting

$$
p(\phi)=\sum_{n=1}^{\infty} 2^{-n} \frac{\|\phi\|_{n}}{1+\|\phi\|_{n}} \quad\left(\phi \in \mathcal{O}_{A}\right)
$$

for example, we obtain a quasinorm on $\mathcal{O}_{A}$ under which $\mathcal{O}_{A}$ becomes a separated locally convex space with a countable base of neighbourhoods of zero, therefore metrizable, but in general not complete; we will denote this space by $\left(\mathcal{O}_{A}, p\right)$.

We now show that on $\mathcal{O}_{A}$ the locally convex topology $\tau^{*}$ of the $H$-limit of the Hausdorff spectrum $\mathcal{X}(\mathcal{S})$ is not weaker than $p$. In fact, let $W=\{\phi \in$ $\left.\mathcal{O}_{A}:\|\phi\|_{N}<\epsilon\right\}$ be some neighbourhood of zero in $\left(\mathcal{O}_{A}, p\right)$ and let $F \in \mathbf{F}$. Let us choose $s_{0} \in|F|$ such that $U_{s_{0}} \supset K_{N}$ - this choice turns out to be possible because of the compactness of $K_{N}$ and the condition $A \subset \bigcup_{F} U_{s}$; also we can find a compact set $K_{m}^{0} \subset U_{s_{0}}$ such that $K_{m}^{0} \supset K_{N}$ - here the choice is possible because of the availability of a fundamental system $\left\{K_{n}^{0}\right\}_{n=1}^{\infty}$ in $U_{s_{0}}$. Now it is clear that

$$
\mathcal{H} \circ \psi\left(M^{F}\right) \subset W
$$

where

$$
\xi=\left(f_{s}\right)_{F}, M^{F}=\left\{\xi \in V_{F}^{s_{0}}: \sup _{z \in K_{m}^{0}}\left|f_{s_{0}}(z)\right|<\epsilon\right\}, \mathcal{H} \circ \psi(\xi)=\phi,\left.f_{s_{0}}\right|_{A}=\phi .
$$

Since $\psi\left(M^{F}\right)$ is itself a neighbourhood of zero in the MVG $X_{(F)}$ and $F \in \mathbf{F}$ was chosen arbitrarily, we have that

$$
\mathcal{H}\left(\operatorname{co}_{\mathbf{F}} \psi\left(M^{F}\right)\right) \subset W
$$

and is a neighbourhood of zero in the topology $\tau^{*}$. This also shows that $\tau^{*} \geq$ $p$.

The conditions of Theorem 3.1 are satisfied, for example, by $A=\bar{\Delta}(0, r)$, the compact polydisk in $\mathbb{C}^{n}$, or by any domain $\mathcal{D} \subset \mathbb{C}^{n}$. It is not difficult to see that if $A$ is a connected set and $A=\bigcap_{\mathbf{F}} \bigcup_{F} U_{s}$, where $\mathbf{F}$ is an admissible class for $\Omega$, then without loss of generality we may assume that the $U_{s}(s \in|\mathbf{F}|)$ are connected open sets in $\mathbb{C}^{n}$. In fact, let each $U_{s}$ have nonempty intersection with $A$, which is natural and can always be arranged by the method of transformation of indices $(s \in|\mathbf{F}|)$. Let us denote by $\widetilde{U}_{s}$ the open connected component of $U_{s}$ which contains $U_{s} \cap A(s \in|\mathbf{F}|)$. Now it is clear that $A=\bigcap_{\mathbf{F}} \bigcup_{F} \widetilde{U}_{s}$ for the admissible class $\mathbf{F}$ in $\Omega$ and moreover, if $\left\{\bigcup_{F} U_{s}\right\}_{\mathbf{F}}$ were a fundamental system of 
neighbourhoods for $A$, then $\left\{\bigcup_{F} \widetilde{U}_{s}\right\}_{\mathbf{F}}$ would be the same. Now let us consider the question: For what classes of sets $A \subset \mathbb{C}^{n}$ do we have a representation

$$
A=\bigcap_{F \in \mathbf{F}} \bigcup_{s \in F} U_{s}
$$

where $\mathbf{F}$ is an admissible class for $\Omega$ (a countable set)?

Let $A$ be any nonempty bounded connected subset of $\mathbb{C}^{n}$ and $B=B\left(z_{0}, r\right)$ an open ball such that $\bar{A} \subset B$. For the $s$-set $B \backslash A$ we have the representation

$$
B \backslash A=\bigcup \bigcup_{\widehat{B} \in \mathcal{K}} \bigcap_{t \in \widehat{B}} L_{t},
$$

where the $L_{t}$ are open subsets of $B\left(z_{0}, r\right)(t \in|\mathcal{K}|,|\mathcal{K}|$ a countable set) and $\mathcal{K}$ is some family of subsets $\widehat{B} \subset \Omega$; moreover, for each $\widehat{B} \in \mathcal{K}$ the intersections $\left\{\bigcap_{\widehat{B}} L_{t}\right\}_{\mathcal{K}}$ form a fundamental system of compact subsets of $B \backslash A$. Since $\mathbb{C}^{n}$ is a finite-dimensional space, we will obtain the representation

$$
B \backslash A=\bigcup_{\widehat{B} \in \mathcal{K}} \bigcap_{t \in \widehat{B}} \bar{L}_{t},
$$

where the $\bar{L}_{t} \subset B\left(z_{0}, r+\epsilon\right)$ are compact sets $(t \in|\mathcal{K}|)$. Now

$$
A=B \backslash \bigcup_{\mathcal{K}} \bigcap_{\widehat{B}} \bar{L}_{t}=\bigcap_{\mathcal{K}}\left(B \backslash \bigcap_{\widehat{B}} \bar{L}_{t}\right)=\bigcap_{\mathcal{K}} \bigcup_{\widehat{B}}\left(B \backslash \bar{L}_{t}\right),
$$

and $G_{t}=B \backslash \bar{L}_{t}$ is an open set in $\mathbb{C}^{n}(t \in|\mathcal{K}|)$. We will show that $\left\{\bigcup_{\widehat{B}} \widetilde{G}_{t}\right\}_{\mathcal{K}}$ form a fundamental system of open connected neighbourhoods of $A$. In fact, if $W \supset A$, $W \subset B$ is an open set, then without loss of generality we may assume that $W \subset B\left(z_{0}, r-\delta\right)$ for some $\delta>0$, so that $P=\left(B\left(z_{0}, r-\delta\right) \cup \partial B\right) \backslash W$ is a compact subset of $B\left(z_{0}, r\right)$. Therefore there exists a compact subset $\bigcap_{\widehat{B}_{0}} \bar{L}_{t}=\bigcap_{\widehat{B}_{0}} L_{t}$ such that $P \subset \bigcap_{\widehat{B}_{0}} \bar{L}_{t}$ and, consequently,

$$
B \backslash P \supset \bigcup_{\widehat{B}_{0}}\left(B \backslash \bar{L}_{t}\right) \quad \text { or } \quad W \cup\left\{z: r-\delta<\left|z-z_{0}\right|<r\right\} \supset \bigcup_{\widehat{B}_{0}} G_{t} \supset \bigcup_{\widehat{B}_{0}} \widetilde{G}_{t} .
$$

However, because of the connectedness of $\widetilde{G}_{t}$ and the ordering of $\widehat{B}_{0} \in \mathcal{K}$ we obtain the inclusion $W \supset \bigcup_{\widehat{B}_{0}} \widetilde{G}_{t}$, which was to be established.

Thus we obtain the following

Theorem 3.3. Every connected bounded subset $A \subset \mathbb{C}^{n}$ has a representation

$$
A=\bigcap_{F \in \boldsymbol{F}} \bigcup_{s \in F} U_{s}
$$

where $\boldsymbol{F}$ is an admissible class for the countable set $\Omega$ and the $U_{s}$ are connected open subsets (domains) in $\mathbb{C}^{n}$.

In particular, for such a set $A$ the Hausdorff spectrum

$$
\mathcal{X}(\mathcal{S})=\left\{\Gamma\left(U_{s}, \mathcal{S}\right), \mathbf{F}, \rho_{U_{s^{\prime}} U_{s}}\right\}
$$

is true (it suffices to apply the uniqueness Theorem for holomorphic functions). In the representation (3.1) it is natural to require that if $U_{s} \cap U_{s^{\prime}} \neq \emptyset\left(s, s^{\prime} \in|\mathbf{F}|\right)$ then it is a connected set. 
In what follows the space $\mathcal{O}_{A}$ of germs of holomorphic functions on $A$ will be provided with the topology $p$ (in general not separated) of uniform convergence on the compact subsets of $A$ and with the locally convex topology of the $H$-limit. As has already been noted above (Theorem 3.1), for a connected bounded subset $A \subset \mathbb{C}^{n}$ we have the linear isomorphism

$$
X \equiv \Gamma(A, \mathcal{S}) \equiv \mathcal{O}_{A} .
$$

We also note that if the set $A$ has an interior point then $\mathcal{O}_{A}$ coincides with the space of holomorphic functions on $A$ (up to isomorphism).

\section{References}

[1] U. Bruzzo, D. G. Markushevich, A. S. Tikhomirov, Symplectic instanton bundles on P3 and 't Hooft instantons, Eur. J. Math. 2:1 (2016), 73-86.

[2] M. De Wilde, Réseaus dans les espaces linéaires à seminormes, Mém. Soc. Royale Sci. Liège 18 (1969) 1-144.

[3] R. C. Gunning, H. Rossi, Analytic functions of several complex variables, Englewood Cliffs, Prentice Hall, New Jersey, 1965.

[4] R. Hartshorne, Stable reflexive sheaves, Math. Ann. 254 (1980), 121-176.

[5] A. Ivanov, A. S. Tikhomirov, Semistable rank 2 sheaves with singularities of mixed dimension on $\mathbb{P}^{3}$, Journal of Geom. and Phys. 129 (2018), 90-98.

[6] M. Jardim, M. Maican, A. Tikhomirov, Moduli spaces of rank 2 instanton sheaves on the projective space, Pacific J. Math. 291:2 (2017), 399-424.

[7] M. Jardim, D. Markushevich, A. S. Tikhomirov, Two infinite series of moduli spaces of rank 2 sheaves on $\mathbb{P}^{3}$, Annali di Mat. Pura ed Appl. 196:4, (2017), 1573-1608.

[8] M. Jardim, D. Markushevich, A. S. Tikhomirov, New divisors in the boundary of the instanton moduli space, Moscow Math. J. 18:1 (2018), 117-148.

[9] A. A. Kytmanov, A. S. Tikhomirov, S. A. Tikhomirov, Series of rational moduli components of stable rank two vector bundles on $\mathbb{P}^{3}$, Selecta Math. (N. S.) 25:29 (2019).

[10] A. A. Kytmanov, N. N. Osipov, S. A. Tikhomirov, Finding Ein components in the moduli spaces of stable rank 2 bundles on the projective 3 -space, Siberian Math. J. $\mathbf{5 7 : 2}(2016), 322-329$.

[11] N. N. Osipov, S. A. Tikhomirov, On the Number of Vedernikov-Ein irreducible components of the moduli space of Stable Rank 2 Bundles on the Projective Space, Siberian Math. J. 59:1 (2018), 107-112.

[12] V. P. Palamodov, Functor of projective limit in the category of topological linear spaces, Math. Collect. 75:4 (1968), 567-603.

[13] V. P. Palamodov, Homological methods in the theory of locally convex spaces, Russian Math. Surveys 26:1 (1971), 1-64.

[14] D. A. Rajkov, On the closed graph theorem for topological linear spaces, Siberian Math. J. 7:2 (1966), 353-372.

[15] E. I. Smirnov, On continuity of semiadditive functional, Math. Notes, 19:4 (1976), $541-548$.

[16] E. I. Smirnov, The theory of Hausdorff spectra in the category of locally convex spaces, Funct. Aprox. 24 (1996), 17-33.

[17] E. I. Smirnov, Hausdorff spectra in functional analysis, Springer, 2002.

[18] A. S. Tikhomirov, S. A. Tikhomirov, D. A. Vassiliev, Construction of Stable Rank 2 Bundles on P3 Via Symplectic Bundles, Siberian Math. J. 60:2 (2019), 343-358.

[19] S. .A. Tikhomirov, Families of stable bundles of rank 2 with $c_{1}=-1$ on the space $\mathbb{P}^{3}$, Siberian Math. J. 55:6 (2014), 1137-1143. 
[20] P. P. Zabreiko, E. I. Smirnov, On the closed graph theorem, Funct. Aprox. 18:2 (1977), 305-316.

Eugeny I. Smirnov

Department of Physics and Mathematics, Yaroslavl State Pedagogical University named after K. D. Ushinskii, Yaroslavl, 150000, Russia

E-mail address: smiei@mail.ru

Sergey A. Tikhomirov

Department of Physics and Mathematics, Yaroslavl State Pedagogical University named after K. D. Ushinskii, Yaroslavl, 150000, Russia

E-mail address: satikhomirov@mail.ru

Elena A. Zubova

Branch of Ural State University of Railway Transport in Tyumen, Tyumen, 625008, Russia

E-mail address: zubovaea@rambler.ru

Received: August 15, 2019; Accepted: December 19, 2019 